\title{
A Complete Census of AGN and Their Hosts from Optical Surveys?
}

\author{
Vivienne Wild ${ }^{1}$, Timothy Heckman ${ }^{2}$, Paule Sonnentrucker $^{2}$, \\ Brent Groves $^{3}$, Lee Armus ${ }^{4}$, David Schiminovich ${ }^{5}$, \\ Benjamin Johnson ${ }^{6}$, Lucimara Martins ${ }^{7}$, and Stephanie LaMassa ${ }^{2}$ \\ ${ }^{1}$ Institut d'Astrophysique de Paris, 75014 Paris, France. \\ Email: wild@iap.fr \\ ${ }^{2}$ Dept. of Physics and Astronomy, Johns Hopkins University, Baltimore, MD 21218, USA \\ ${ }^{3}$ Leiden Observatory, Leiden University, P.O. Box 9513, 2300 RA Leiden, The Netherlands \\ ${ }^{4}$ Caltech, Spitzer Science Center, MS 314-6, Pasadena, CA 91125, USA \\ ${ }^{5}$ Dept. of Astronomy, Columbia University, NewYork, NY10027, USA \\ ${ }^{6}$ Institute of Astronomy, Madingley Road, Cambridge, CB3 0HA, UK \\ ${ }^{7}$ NAT-Universidade Cruzeirodo Sul, Sao Paulo, Brazil
}

\begin{abstract}
Large optical surveys provide an unprecedsCeented census of galaxies in the local Universe, forming an invaluable framework into which more detailed studies of objects can be placed. But how useful are optical surveys for understanding the co-evolution of black holes and galaxies, given their limited wavelength coverage, selection criteria, and depth? Here we present work-in-progress comparing optical and mid-IR diagnostics of three "unusual" low-z populations (luminous Seyferts, dusty Balmer-strong AGN, ULIRGs) with a set of ordinary star-forming galaxies from the SDSS. We address the questions "how well do the mid-IR and optical diagnostics of star formation and AGN strength agree?" and "to what extent do optical surveys allow us to include extreme, dusty, morphologically disturbed galaxies in our 'complete' census of BH-galaxy co-evolution?
\end{abstract}

Keywords. infrared: galaxies, galaxies: active, galaxies: starburst, galaxies: ISM

\section{Introduction}

In the last few decades, large optical spectroscopic surveys have allowed enormous progress to be made in studying the galaxy population, both the overall demographics and the identification of rare populations. The role of AGN on the evolution of the galaxy population has also become an increasingly common topic of research, largely driven by theoretical models and simulations which suggest some additional input of energy into the ISM and IGM is required in order to move galaxies from the blue to the red sequence and prevent star formation in massive galaxies.

At low to intermediate redshifts, the sheer statistics of current optical surveys allow us to identify and study significant numbers of rare objects which represent transient phases in the lifetime of galaxies: starburst, post-starburst and "green-valley" galaxies, major mergers, and powerful AGN. These brief periods of major disruption in the life of a galaxy may be insignificant compared to a typical galaxy's total life span, but hugely significant globally, i.e., when the effects are integrated over the whole population and the age of the Universe. For example, summing the growth of black holes attained during rare, high growth-rate phases (high $L / L_{\mathrm{Edd}}$ ) gives a black hole mass density close to that observed in the local Universe, implying that lower growth-rate events are relatively unimportant for building black holes (Yu \& Tremaine 2002; Heckman et al. 2004). 
Optical surveys allow us to locate sufficient samples of galaxies experiencing transient phases of evolution. There is an obvious benefit in using the information provided in the detection survey to study the galaxies in greater detail, but, with the complex physical processes at play during these periods of disruption, it is uncertain how far optical studies alone can help us in understanding the true impact of these transient phases on the lifecycle of the galaxy. How much physical information can we really infer from the optical when faced with extreme star formation, dust production, AGN activity, and complicated spatial geometries of disturbed galaxies?

Here, we present ongoing work comparing star formation and AGN properties derived from mid-infra-red spectra from the Spitzer space telescope, to those derived from optical spectra. We combine information from four surveys, three of which probe an "unusual" phase in the life of a galaxy and one control sample of "ordinary" galaxies.

\section{Samples}

SSGSS: The control sample is the Spitzer-SDSS-GALEX Spectroscopic Survey (SSGSS, see O'Dowd et al. 2009), a representative sample of 100 local galaxies selected from the Sloan Digital Sky Survey (SDSS) with GALEX photometry for follow-up spectroscopy with the Spitzer-IRS. Their mass, color, star formation rate, and redshift distribution are representative of galaxies in the SDSS survey.

Dusty Balmer-strong: These galaxies were selected from the SDSS-DR4 to be candidate "post-starburst" AGN with strong Balmer absorption lines. They also possess very high dust contents that may mean the strong Balmer absorption lines are caused by dust geometry rather than star formation history (Poggianti \& Wu 2000).

ULIRGs: These are the 10 ULIRGs in the IRAS Bright Galaxy Sample; the Spitzer IRS low- and high-resolution observations of these galaxies have been presented in detail by Armus et al. (2007). Of the 10 BGS ULIRGs, six are found in the SDSS spectroscopic sample, three do not lie within the SDSS footprint, and one is too faint to have been targeted.

Seyferts: The narrow-line Seyfert sample is composed of the top 20 [O III] $\lambda 5007$ flux emitters out of all SDSS-DR4 main-sample galaxies classified as Seyfert galaxies using the diagnostic line ratio plot of $[\mathrm{N}$ II $] / \mathrm{H} \alpha$ vs. $[\mathrm{O}$ III $] / \mathrm{H} \beta$.
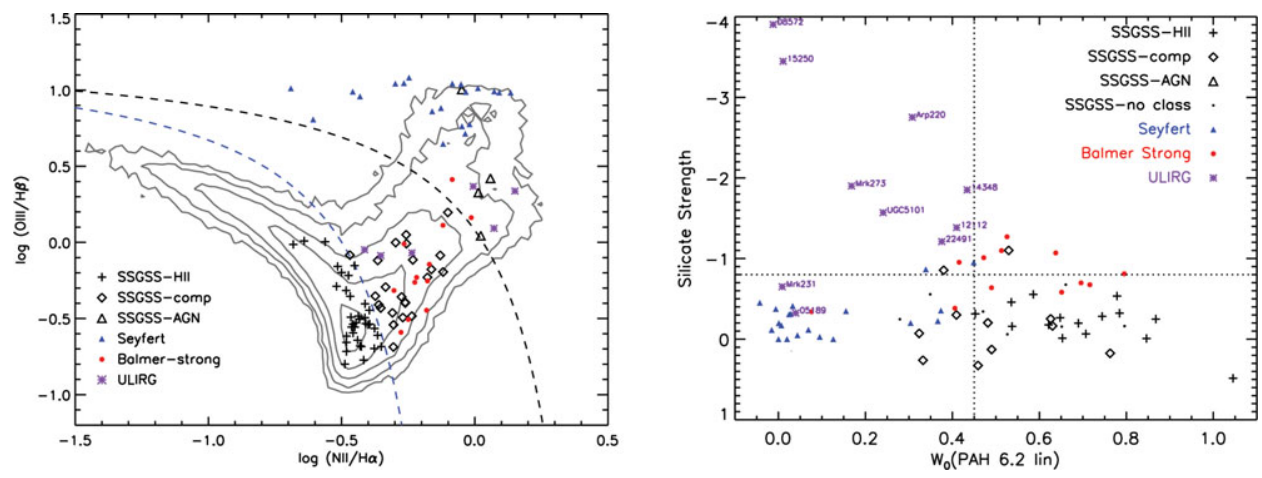

Figure 1. Left: The optical BPT diagram of nebular emission-line ratios. Only those galaxies with signal-to-noise ratio $S / N>3$ in all four emission lines are plotted. Right: The Spoon et al. (2007) IR diagnostic diagram. Only those galaxies with mean per-pixel $S / N>4$ in the wavelength range $5.4-5.9 \mu \mathrm{m}$ are plotted. Formal upper limits on $W_{6.2}$ are not calculated: $W_{6.2}$ values $\lesssim 0.05$ should be considered consistent with zero. 
In Figure 1, we place the four samples into context, for both optical and IR astronomers. In the left-hand panel, we show the BPT diagram of optical emission-line ratios that separates galaxies with nebular emission dominated by star formation (left-hand branch) from those dominated by higher ionization processes occurring in the galactic nucleus (right-hand branch). The simplest explanation for the population that lies between the two demarcation lines is that both star formation and an AGN are present ("compositeAGN"). This is supported by their younger mean stellar ages as measured from the stellar continuum, compared to galaxies with "pure-AGN" emission line ratios (Kauffmann et al. 2003; Kewley et al. 2006). The gray contours indicate the joint distribution of all SDSSDR7 galaxies, the symbols show the four samples described above. Both the dustyBalmer strong galaxies and the Seyferts were pre-selected to lie in the AGN branch. All six ULIRGs, selected purely on IR luminosity, show optical emission-line ratios which indicate the presence of an obscured AGN. In the right-hand panel of Figure 1 we place all our samples onto the diagram of Spoon et al. (2007) which compares the rest frame equivalent width of the $6.2 \mu \mathrm{m}$ PAH feature caused by on-going star formation, with the strength of the silicate absorption feature at $10 \mu \mathrm{m}$ caused by dust absorbing the mid-IR continuum along the line of sight (see following section). As expected from their optical classification as AGN, both the Seyfert sample and the SSGSS composite-AGN have on average lower $W_{6.2}$ than ordinary star-forming galaxies, indicative of the destruction of small grain PAHs in the strong radiation field of the QSO. The Balmer-strong AGN show stronger silicate absorption than ordinary galaxies, once again qualitatively consistent with their large Balmer decrements ( $\mathrm{H} \alpha$ to $\mathrm{H} \beta$ emission-line ratios) in the optical.

\section{Dust}

The effect of dust grains intercepting stellar and nuclear light complicates the analysis of integrated galaxy spectra. In the optical, extinction of the light along the line-of-sight can be quantified through a measured Balmer decrement ( $\mathrm{H} \alpha$ to $\mathrm{H} \beta$ emission line ratio), combined with an empirically measured dust extinction curve (i.e. the Milky Way, LMC or SMC). However, the additional scattered component that makes up a full attenuation curve important for studies of integrated galaxy light has only been measured empirically for the continua of starburst galaxies (Calzetti et al. 1994). Emission lines are expected to suffer more screenlike (less grey) attenuation than the optical continua, as the emission must first pass through the dense birth clouds in which the hot $\mathrm{O}$ and $\mathrm{B}$ stars that excite the nebular emission lines form (Charlot \& Fall 2000; Wild et al. 2007; da Cunha et al. 2008). An additional complication arises in the case of composite galaxies, in which the line emission originating from the narrow line region of the AGN may suffer a different level and/or form of extinction compared to nebular emission originating in star-forming regions.

Extinction in the mid-IR is dominated by features caused by the stretching and bending modes of amorphous silicate grains. The presence of silicate absorption can be used to infer the geometry of material obscuring the light source (Levenson et al. 2007; Nenkova et al. 2002).

In Figure 2, we compare two different measures of dust content in the IR and optical regimes. Aside from the two extreme ULIRGs, there is a general trend for the two measures of dust content to track one another. Although both axes describe dust content, the trend is slightly surprising: any significant silicate absorption applies optically thick clouds $\left(\tau_{V}>100\right)$, which contrasts strongly with the $\tau_{V}$ measured here in the optical. Therefore these two quantities measure dust in entirely independent regions. It is interesting to note that the two outliers from the trend (ULIRGs 15250 and 08572), 


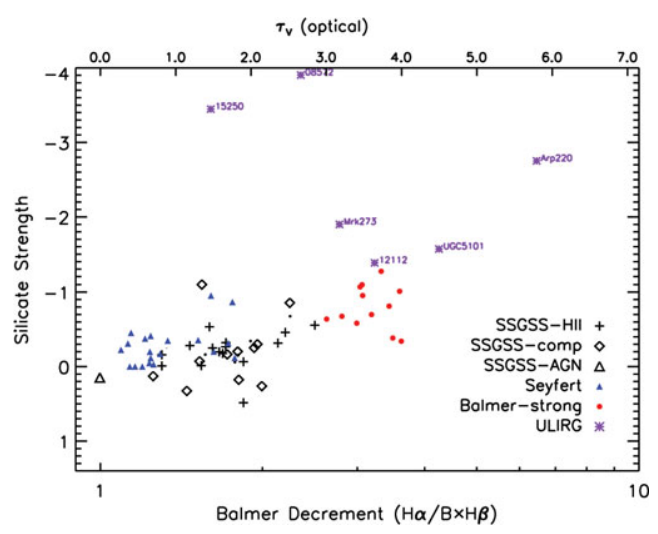

Figure 2. Comparing dust obscuration in the IR (10 $\mu \mathrm{m}$ silicate absorption) to dust obscuration in the optical (the Balmer decrement). We set $B=2.87$ (Case B recombination) for star forming and composite galaxies, and $B=3.1$ for pure-AGN. Only those galaxies with mean per-pixel $S / N>4$ in the wavelength range $5.4-5.9 \mu \mathrm{m}$, and $S / N>3$ in their $\mathrm{H} \alpha$ and $\mathrm{H} \beta$ lines are plotted. The upper axis gives $\tau_{V}$, derived from the Balmer decrement assuming the two-component Charlot and Fall (2000) dust attenuation curve with $n_{\mathrm{BC}}=-1.3$ and $n_{\mathrm{ISM}}=-0.7$ (Wild et al. 2007).

with extremely strong silicate absorption implying that the source of the mid-IR continuum is buried in a smooth, geometrically and optically thick cloud, are composite-AGN in the optical. Therefore, some light from the central nucleus is escaping to illuminate the narrow-line region, or the AGN is not the dominant light source responsible for the mid-IR continuum and the dust is not associated with the AGN torus.

\section{Low-Ionization Emission Lines}

In star-forming galaxies, the dominant excitation mechanism for nebular emission lines is UV radiation from young, hot stars. When star formation is the dominant mechanism of excitation, $\mathrm{H} \alpha$ is one of the best calibrated measures of instantaneous star formation rate in galaxies. However, because the $\mathrm{H} \alpha$ line lies in the optical, to obtain accurate SFR estimates we must correct for dust attenuation. In this section we address the question of how accurate the standard methods of correction for dust attenuation in the optical are, by comparing the $\mathrm{H} \alpha$ luminosity to the luminosity of the lower ionization neon lines in the mid-IR. To first order, the strengths of these lines are expected to be strongly correlated, especially in the relatively massive, and therefore metal-rich, galaxies that make up the majority of our samples.

In Figure 3, we plot $\mathrm{H} \alpha$ vs. Ne luminosities for all of the samples, firstly with $\mathrm{H} \alpha$ uncorrected for dust attenuation, then corrected with two different dust attenuation laws used in the literature. In the top-left panel, where no correction for dust attenuation has been applied to the $\mathrm{H} \alpha$ luminosities, we immediately notice that the Balmer-strong galaxies and ULIRGs lie above the SSGSS galaxies as expected for their large dust contents. In the top-right panel, the dust law is a single component model with $\tau \propto \lambda^{-0.7}$. This law provides a good fit to the UV and IR continua of starburst galaxies (Charlot \& Fall 2000, hereafter CF00), but we see that it does not provide a good fit to the nebular emission lines, causing considerable overcorrection of the optical emission lines in the dustiest galaxies and undercorrection in the least dusty Seyferts compared to normal SSGSS-H II galaxies. In the bottom-left panel, we employ the full two-component dust model of CF00, where the second term allows for the additional, greyer, attenuation of 

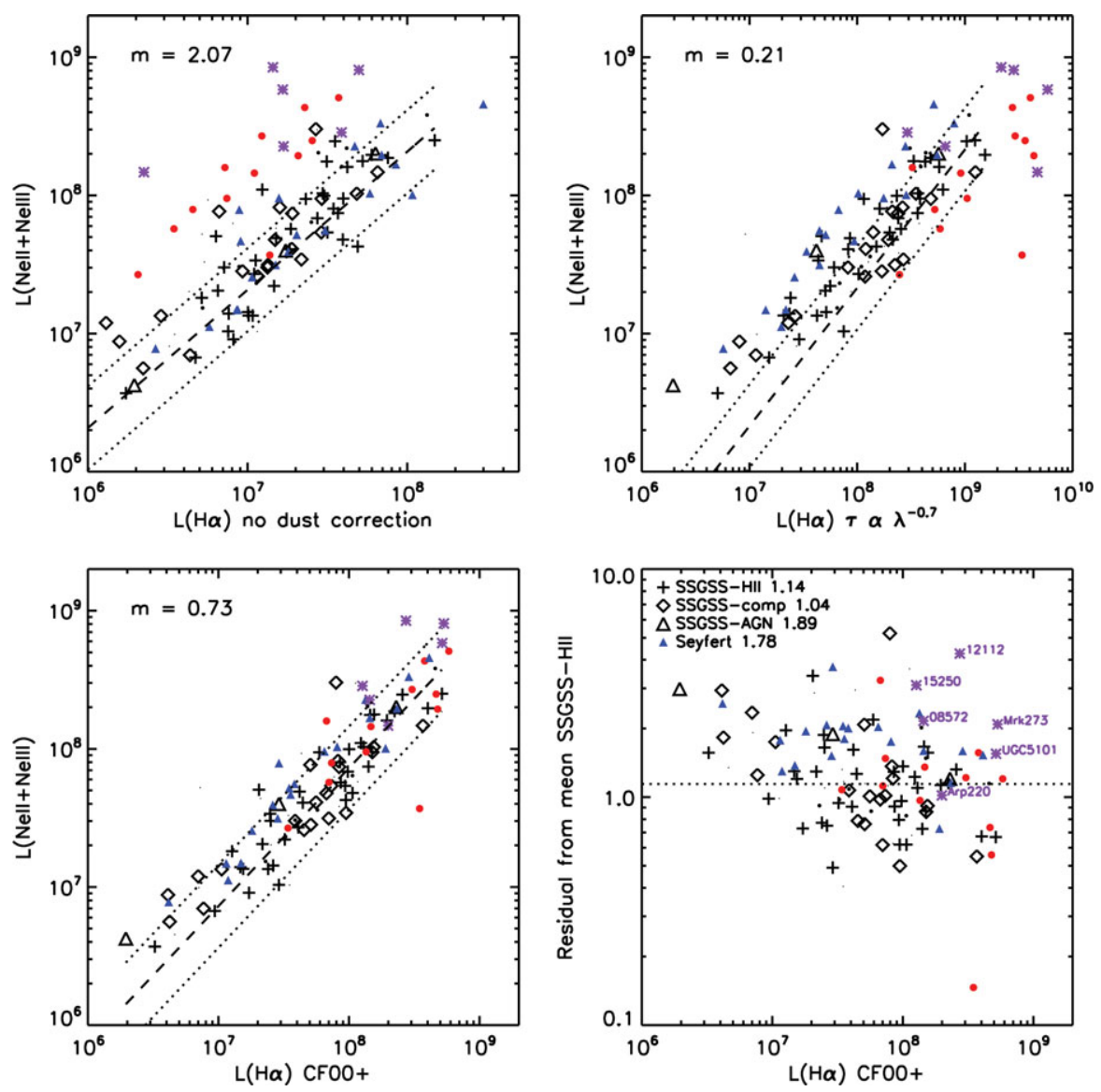

Figure 3. The luminosity of $\mathrm{H} \alpha$ in the optical vs. the low ionization Ne lines in the mid-IR. Top left: $\mathrm{H} \alpha$ is not corrected for dust attenuation. Top right: $\mathrm{H} \alpha$ is corrected for dust attenuation using a single component CF00 model. Bottom left: $\mathrm{H} \alpha$ is corrected for dust attenuation using a double component CF00 model. In all three panels, the dashed line shows the best-fit linear fit to the SSGSS star-forming galaxies, and the dotted lines show a factor of two above and below. Bottom right: The residual from the linear fit in the bottom-left panel.

the stellar birth clouds with an exponent of -1.3 (Wild et al. 2007; da Cunha et al. 2008). There is a noticeable improvement in the scatter. In each panel, the dashed line shows a linear least-squares fit to the SSGSS-H II galaxies, with the dotted lines indicating a factor of two above and below the best-fit. In the bottom-right we plot the residual away from this best-fit line using the two-component CF00 model. The scatter is around a factor of two, even for the extreme dusty Balmer-strong galaxies. Even the ULIRGs do not show a dramatic underprediction of their $\mathrm{H} \alpha$ luminosities, despite their optical Balmer decrements indicating as much as six magnitudes of extinction and their silicate absorption indicating hundreds of magnitudes. We note a slight mean offset for the AGN, in the sense that they have stronger Ne II+Ne III emission for their $\mathrm{H} \alpha$ luminosities. This could be due to the harder ionizing radiation field of the AGN, or that the true dust attenuation law is slightly different in the narrow line region of the AGN. Although the effect is not large, further study may be able to untangle these two effects. 


\section{High-Ionization Emission Lines}

Given the success of the 2 component dust law for correcting $\mathrm{H} \alpha$ for dust attenuation in even the dustiest galaxies in the local Universe, we now turn to the question of using [O III] luminosity as a proxy for black hole accretion rate. Although originating from the narrow line region, far removed spatially from the central nucleus with its dense obscuring torus, the [O III] emission must still suffer extinction from the host galaxy, and possibly also from dust within the narrow line region. This adds an additional degree of complexity to the problem: the $\mathrm{H} \alpha$ and $\mathrm{H} \beta$ emission lines with which we calculate the magnitude of the dust attenuation in the optical, may originate at least in part from star-forming regions completely spatially separated from the narrow-line region in which we are interested. However, given the small residual offset between $\mathrm{Ne}$ and $\mathrm{H} \alpha$ emission presented in the previous section, even for the strongest AGN in the SDSS (the Seyfert sample), this effect cannot be large.

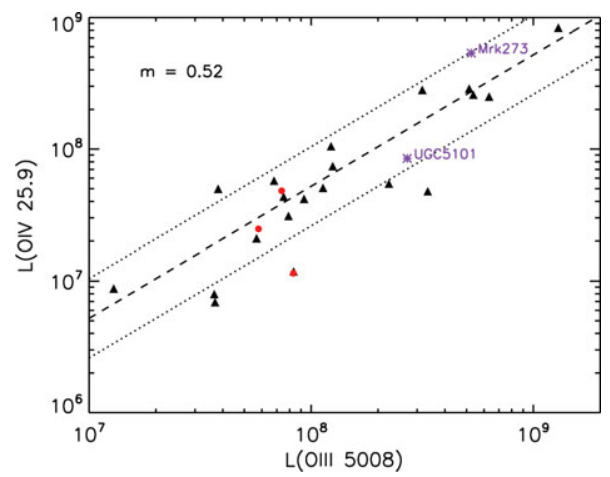

Figure 4. Dust-corrected $L([\mathrm{O} I I I])$ vs. $L([\mathrm{O} I V])$ for the Seyfert sample (filled triangles), the three Balmer-strong galaxies with [O IV] detections (circles) and the two ULIRGs with both [O IV] detections and SDSS spectra.

In Figure 4, we plot individual detections of [O IV] $\lambda 25.9 \mu \mathrm{m}$ versus [O III] $\lambda 5007$, using the Balmer decrement and two component CF00 model to correct [O III] for dust attenuation. The mid-IR [O IV] line lies too close to an Fe II line for deconvolution in the low-resolution Spitzer spectra. Therefore we only plot the Seyfert, ULIRG, and dusty Balmer-strong galaxy samples, for which we have high resolution spectra. Of the galaxies in this figure, two of the Balmer-strong galaxies and both of the ULIRGs are optically classified as pure-AGN, and the majority of the [O III] is therefore likely to originate from the AGN rather than from star formation. For the third Balmer-strong galaxy, a small correction has been applied to account for the star formation contamination of the [O III] line. At higher ionization, the [O IV] line is expected to suffer less contamination from star formation.

Once again, we find that where there are individual detections of both [O IV] and [O III], the line luminosities are strongly correlated after appropriate correction for dust attenuation of [O III], even in extremely dusty galaxies. Although further work is required to investigate those galaxies where [O IV] is not detected in the Spitzer spectra, this preliminary result suggests that dust attenuation is not a significant cause for concern in the use of optical spectra for deriving black hole accretion rates. 


\section{Discussion}

In this preliminary investigation comparing the mid-IR and optical emission lines of three samples of unusual galaxies in the local Universe, and a sample of more ordinary galaxies, we find little evidence that the optical wavelength regime, used alone, would provide a misleading picture in terms of either star formation rates or black hole accretion rates. Each of the three samples is extreme in some way: the Seyferts are the strongest [O III] emitters in the local Universe, the Balmer-strong galaxies are some of the dustiest AGN in the SDSS, the ULIRGs are unusually bright in the IR, implying substantial dust contents. We have shown how each of these samples fits into the global galaxy population, both in the mid-IR and in the optical.

It is perhaps surprising that, given the geometrically complicated dust distributions of some galaxies, and the spatial separation between the narrow line region and the starforming regions in composite-AGN, that nebular emission lines in the optical wavelength regime can be adequately corrected for the effects of dust attenuation. With further investigation of these samples, we hope to understand more about the limitations, or otherwise, of the optical wavelength regime for studying unusual galaxies.

\section{References}

Armus, L., et al. 2007, ApJ, 656, 148

Calzetti, D., Kinney A. L., \& Storchi-Bergmann T. 1994, ApJ, 429, 582

Charlot, S. \& Fall, S. M. 2000, ApJ, 539, 718

da Cunha, E., Charlot, S., \& Elbaz, D. 2008, MNRAS, 388, 1595

Heckman, T. M., Kauffmann, G., Brinchmann, J., Charlot, S., Tremonti, C., \& White, S. D. M. 2004, ApJ, 613, 109

Kauffmann, G., et al. 2003, MNRAS, 346, 1055

Kewley, L. J., Groves, B., Kauffmann, G., \& Heckman, T. 2006, MNRAS, 372, 961

Levenson, N. A., Sirocky, M. M., Hao, L., Spoon, H. W. W., Marshall, J. A., Elitzur, M., \& Houck, J. R. 2007, ApJ, 654, L45

Nenkova, M., Ivezić, Ž., \& Elitzur, M. 2002, ApJ, 570, L9

O'Dowd, M. J., et al. 2009, ApJ, 705, 885

Poggianti, B. M. \& Wu, H. 2000, ApJ, 529, 157

Spoon, H. W. W., Marshall, J. A., Houck, J. R., Elitzur, M., Hao, L., Armus, L., Brandl, B. R., \& Charmandaris, V. 2007, ApJ, 654, L49

Wild, V., Kauffmann, G., Heckman, T., Charlot, S., Lemson, G., Brinchmann, J., Reichard, T., \& Pasquali A. 2007, MNRAS, 381, 543

Yu, Q. \& Tremaine, S. 2002, MNRAS, 335, 965 